\title{
Green's Functions and DOS for Some 2D Lattices
}

\author{
Eugene Kogan ${ }^{1,2}$, Godfrey Gumbs ${ }^{3}$ \\ ${ }^{1}$ Department of Physics, Bar-Ilan University, Ramat-Gan, Israel \\ ${ }^{2}$ Max-Planck-Institut für Physik Komplexer Systeme, Dresden, Germany \\ ${ }^{3}$ Department of Physics and Astronomy, Hunter College of the City University of New York, New York, NY, USA \\ Email: Eugene.Kogan@biu.ac.il, ggumbs@hunter.cuny.edu
}

How to cite this paper: Kogan, E. and Gumbs, G. (2021) Green's Functions and DOS for Some 2D Lattices. Graphene, 10, $1-12$.

https://doi.org/10.4236/graphene.2021.1010 $\underline{01}$

Received: August 23, 2020

Accepted: November 29, 2020

Published: December 2, 2020

Copyright (c) 2021 by author(s) and Scientific Research Publishing Inc. This work is licensed under the Creative Commons Attribution International License (CC BY 4.0).

http://creativecommons.org/licenses/by/4.0/

\begin{abstract}
In this presentation we present the Green's functions and density of states for the most frequently encountered 2D lattices: square, triangular, honeycomb, kagome, and Lieb lattice. Though the results are well known, we hope that its derivation performed in a uniform way provides some pedagogical value.
\end{abstract}

Keywords

2D Lattices

\section{Introduction}

Fermionic lattice models are widely used not only as a purely theoretical tool but also as a basis for investigation and modelling of physical properties of real materials [1]. Despite their relative formal simplicity-the Hamiltonians of many of them can be written down as bilinears of fermionic operators-analytical calculation of the lattice Green's functions can present substantial difficulties.

In general, the lattice Green's functions of systems are ubiquitous [2] [3] [4] in solid state physics, appearing in problems of lattice vibrations, spin wave theory of magnetic systems, localized oscillation modes at lattice defects, combinatorial problems in lattices [5], and flux calculations in lattice percolation [6]. Lattice Green's functions are also central to the theory of random walks on a lattice [7] [8], and to the calculation of the effective resistance of resistor networks [9].

The lattice Green's functions are of central importance for understanding the electronic behavior of perfect crystalline solids. It also provides the basis for understanding the electronic properties of real, imperfect crystalline solids, since 
the imperfections can be treated as a perturbation. Lattice Green's functions are also important for calculating RKKY interaction [10] [11] [12] [13].

We will now consider several popular 2D lattices calculate Green's functions and density of states (DOS). Yielding the same results, the derivation of these results performed in a uniform way and being presented in one place delivers some pedagogical value.

In all cases we'll consider the models with the nearest neighbour hopping only, the amplitude of the hopping we'll take to be 1 , so the Hamiltonian will be

$$
H=\sum_{\langle i j\rangle} c_{i}^{\dagger} c_{j}
$$

where $c^{\dagger}$ and $c$ are electron creation and annihilation operators, and the summation in Equation (1) is with respect to nearest neighbor pairs. The period of the lattice we'll also take to be equal to 1 .

\section{Square Lattice}

In the wave vector representation, the Hamiltonian for the square lattice is

$$
H_{\square}(\boldsymbol{k})=\epsilon_{\square}(\boldsymbol{k})=2 \cos k_{x}+2 \cos k_{y} .
$$

The site diagonal matrix element of the Green's function is

$$
\begin{aligned}
g_{\square}(E) & =\frac{1}{(2 \pi)^{2}} \int_{-\pi}^{\pi} \int_{-\pi}^{\pi} \frac{\mathrm{d} k_{x} \mathrm{~d} k_{y}}{E-\epsilon_{\square}(\boldsymbol{k})} \\
& =\frac{1}{(2 \pi)^{2}} \int_{-\pi}^{\pi} \int_{-\pi}^{\pi} \frac{\mathrm{d} k_{x} \mathrm{~d} k_{y}}{E-2 \cos k_{x}-2 \cos k_{y}} .
\end{aligned}
$$

Employing the identity

$$
\frac{1}{2 \pi} \int_{-\pi}^{\pi} \frac{\mathrm{d} \theta}{a+b \cos \theta}=\frac{\operatorname{sign}(a)}{\sqrt{a^{2}-b^{2}}} \quad(|a|>|b|)
$$

to perform integration with respect to $k_{y}$, we obtain for $E<-4$ or $E>4$

$$
\begin{aligned}
g_{\square}(E) & =\frac{1}{2 \pi} \int_{-\pi}^{\pi} \frac{\mathrm{d} k_{x}}{\sqrt{\left(E-2 \cos k_{x}\right)^{2}-4}} \\
& =\frac{\operatorname{sign}(E)}{2 \pi} \int_{-1}^{1} \frac{\mathrm{d} x}{\sqrt{\left(1-x^{2}\right)(x-c)(x-d)}},
\end{aligned}
$$

where $c=1+\frac{E}{2}, d=-1+\frac{E}{2}$. For $E<-4$ we may use the identity [14]

$$
\int_{b}^{a} \frac{\mathrm{d} x}{\sqrt{(a-x)(x-b)(x-c)(x-d)}}=\frac{2}{\sqrt{(a-c)(b-d)}} K(k),
$$

where $a>b>c>d, K$ is the complete elliptic integral of the first kind, and

$$
k=\sqrt{\frac{(a-b)(c-d)}{(a-c)(b-d)}} .
$$

In our case $a=1, b=-1$; hence we obtain

$$
(a-b)(c-d)=4
$$




$$
(a-c)(b-d)=\frac{E^{2}}{4} \text {. }
$$

Thus we get for $E>4$

$$
g_{\square}(E)=\frac{2}{\pi E} K\left(\frac{4}{E}\right) .
$$

We can analytically continue the Green function from the part of real axis $E<-4$. For $-4<E<4$ we have $k>1$. Hence we should put $E=\epsilon+i 0^{+}$, that is $k$ acquires infinitesimal imaginary part $i 0^{+}$, and we may use the identities [4]

$$
\begin{gathered}
\operatorname{Im}\left[K\left(k+i 0^{+}\right)\right]=-\frac{1}{k} K\left(\sqrt{1-\frac{1}{k^{2}}}\right) \\
\operatorname{Re}\left[K\left(k+i 0^{+}\right)\right]=\frac{1}{k} K\left(\frac{1}{k}\right)
\end{gathered}
$$

to get $[4]$

$$
\rho_{\square}(\epsilon)=-\frac{1}{\pi} \operatorname{Im}\left[g\left(\epsilon+i 0^{+}\right)\right]=\frac{1}{2 \pi^{2}} K\left(\sqrt{1-\frac{\epsilon^{2}}{16}}\right) .
$$

The DOS is presented on Figure 1.

\section{Triangular Lattice}

Let $\boldsymbol{a}_{1}, \boldsymbol{a}_{2}$ and $\boldsymbol{a}_{3}$ be the three periods of the triangular lattice adding up to zero. The Hamiltonian in the wave vector representation is

$$
H_{\Delta}(\boldsymbol{k})=\epsilon_{\Delta}(\boldsymbol{k})=2 \cos k_{1}+2 \cos k_{2}+2 \cos k_{3},
$$

where $k_{i} \equiv \boldsymbol{a}_{i} \cdot \boldsymbol{k}$. The site diagonal matrix element of the Green's function is ( $k_{1}$ and $k_{2}$ are the components of $\boldsymbol{k}$ in the oblique coordinate system)

$$
\begin{aligned}
g_{\Delta}(E) & =\frac{1}{(2 \pi)^{2}} \int_{-\pi}^{\pi} \int_{-\pi}^{\pi} \frac{\mathrm{d} k_{1} \mathrm{~d} k_{2}}{E-\epsilon_{\Delta}(\boldsymbol{k})} \\
& =\frac{1}{(2 \pi)^{2}} \int_{-\pi}^{\pi} \int_{-\pi}^{\pi} \frac{\mathrm{d} k_{1} \mathrm{~d} k_{2}}{E-2 \cos k_{1}-4 \cos \left(k_{2}+\frac{k_{1}}{2}\right) \cos \left(\frac{k_{1}}{2}\right)} .
\end{aligned}
$$

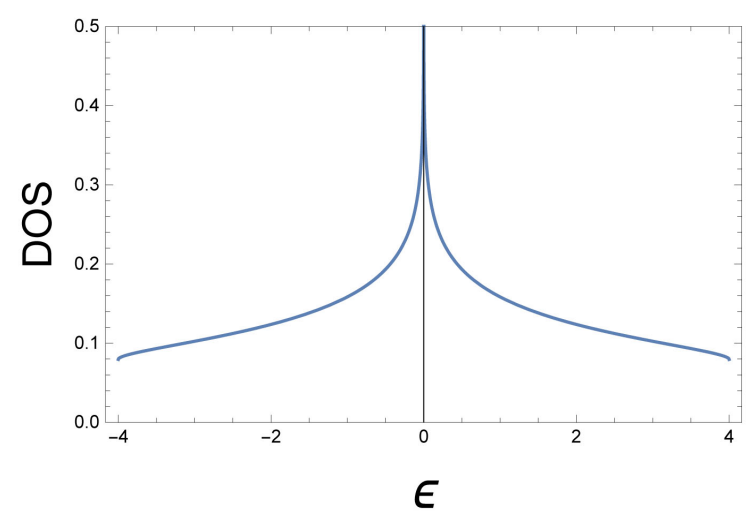

Figure 1. DOS for the square lattice. 
(We used the transformation suggested in Ref. [8]). Employing the identity (4) to perform integration with respect to $k_{2}$ we obtain for $E>6$ or $E<-2$

$$
\begin{aligned}
g_{\Delta}(E) & =\frac{\operatorname{sign}(E)}{2 \pi} \int_{-\pi}^{\pi} \frac{\mathrm{d} k_{1}}{\sqrt{\left(E-2 \cos k_{1}\right)^{2}-8-8 \cos k_{1}}} \\
& =\frac{\operatorname{sign}(E)}{8 \pi} \int_{-\pi}^{\pi} \frac{\mathrm{d} k_{1}}{\sqrt{\left(a-\cos k_{1}\right)\left(b-\cos k_{1}\right)}} \\
& =\frac{\operatorname{sign}(E)}{4 \pi} \int_{-1}^{1} \frac{\mathrm{d} x}{\sqrt{\left(1-x^{2}\right)(a-x)(b-x)}},
\end{aligned}
$$

where [15]

$$
\begin{aligned}
& a=1+\frac{E}{2}+\sqrt{3+E} \\
& b=1+\frac{E}{2}-\sqrt{3+E}
\end{aligned}
$$

For $E>6$ we may use the identity [14]

$$
\int_{d}^{c} \frac{\mathrm{d} x}{\sqrt{(a-x)(b-x)(c-x)(x-d)}}=\frac{2}{\sqrt{(a-c)(b-d)}} K(k),
$$

where $a>b>c>d$, and

$$
k=\sqrt{\frac{(a-b)(c-d)}{(a-c)(b-d)}} .
$$

From Equation (16) follows

$$
\begin{gathered}
(a-b)(c-d)=4 r \\
(a-c)(b-d)=\frac{1}{4}(r-1)^{3}(r+3),
\end{gathered}
$$

where

$$
r=\sqrt{3+E} .
$$

Thus we get for $E>6$ [16] [17]

$$
g_{\Delta}(E)=\frac{1}{\pi(r-1)^{3 / 2}(r+3)^{1 / 2}} K\left(\frac{4 r^{1 / 2}}{(r-1)^{3 / 2}(r+3)^{1 / 2}}\right)
$$

We can analytically continue the Green function from the part of real axis $E>6$. For $-2<E<6$ we have $k>1$. Hence we should put $E=\epsilon+i 0^{+}$, that is $k$ acquires infinitesimal imaginary part $i 0^{+}$, we may use the identities (10), (11) and take into account that

$$
1-\frac{1}{k^{2}}=\frac{(3-r)(r+1)^{3}}{16 r} .
$$

For $-3<E<2$ the value of $k$ become imaginary. We may use the identity [18]

$$
K(i k)=\kappa^{\prime} K(\kappa),
$$

where 


$$
\kappa=\frac{k}{\sqrt{k^{2}+1}}, \quad \kappa^{\prime}=\frac{1}{\sqrt{k^{2}+1}} .
$$

In our case

$$
\kappa=\sqrt{\frac{4 r}{(3-r)(r+1)^{3 / 4}}},
$$

and we reproduce DOS for the triangular lattice [16]

$$
\rho_{\Delta}(\epsilon)=-\frac{1}{\pi} \operatorname{Im}\left[g\left(\epsilon+i 0^{+}\right)\right]=\frac{1}{\pi^{2} \sqrt{z_{0}}} K\left(\sqrt{\frac{z_{1}}{z_{0}}}\right),
$$

where

$$
\begin{aligned}
& z_{0}= \begin{cases}\frac{(3-r)(r+1)^{3}}{4} & \text { for }-3 \leq \epsilon \leq-2 \\
4 r & \text { for }-2 \leq \epsilon \leq 6\end{cases} \\
& z_{1}= \begin{cases}4 r & \text { for }-3 \leq \epsilon \leq-2 \\
\frac{(3-r)(r+1)^{3}}{4} & \text { for }-2 \leq \epsilon \leq 6\end{cases}
\end{aligned}
$$

The DOS is presented on Figure 2.

\section{Honeycomb Lattice}

The honeycomb lattice can be considered as a triangular lattice with a basis of two lattice points. The tight binding Hamiltonian for the electrons is

$$
\hat{H}=\sum_{n} a_{n}^{\dagger}\left(b_{n+\delta_{1}}+b_{n+\delta_{2}}+b_{n+\delta_{3}}\right)+\text { H.c, }
$$

where $\boldsymbol{\delta}_{1}, \boldsymbol{\delta}_{2}, \boldsymbol{\delta}_{3}$ are the vectors connecting an atom with its nearest neighbors.

Going to wave vector representation we obtain

$$
\hat{H}=\sum_{\boldsymbol{k}} \Psi_{\boldsymbol{k}}^{\dagger} \hat{H}(\boldsymbol{k}) \Psi_{\boldsymbol{k}},
$$

where

$$
\hat{H}(\boldsymbol{k})=\left(\begin{array}{cc}
0 & \sum_{i} \mathrm{e}^{-i \boldsymbol{k} \cdot \boldsymbol{\delta}_{i}} \\
\sum_{i} \mathrm{e}^{i \boldsymbol{k} \cdot \boldsymbol{\delta}_{i}} & 0
\end{array}\right) .
$$

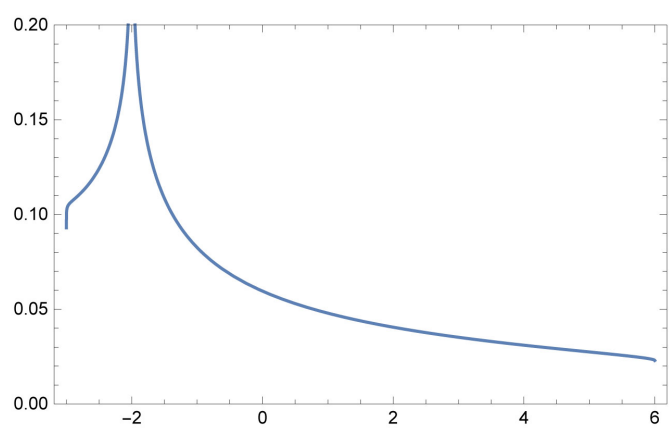

Figure 2. DOS for the triangular lattice. 
Taking into account that $\boldsymbol{\delta}_{1}-\boldsymbol{\delta}_{2}=\boldsymbol{a}_{3}, \boldsymbol{\delta}_{2}-\boldsymbol{\delta}_{3}=\boldsymbol{a}_{1}, \boldsymbol{\delta}_{3}-\boldsymbol{\delta}_{1}=\boldsymbol{a}_{2}$, we obtain the spectrum as

$$
\epsilon_{v}(\boldsymbol{k})=v \sqrt{X_{k}},
$$

where $v= \pm 1$, and

$$
X_{k} \equiv 2\left(\cos k_{1}+\cos k_{2}+\cos k_{3}\right)+3 .
$$

The dispersion law can be written in the form

$$
\epsilon_{v}(\boldsymbol{k})=v \sqrt{3+\epsilon_{\Delta}(\boldsymbol{k})} \text {. }
$$

The Green's function is

$$
g_{H}(E)=\frac{1}{2 \pi^{2}} \int_{-\pi}^{\pi} \mathrm{d} k_{1} \int_{-\pi}^{\pi} \mathrm{d} k_{2} \frac{E}{E^{2}-3-\epsilon_{\Delta}(k)} .
$$

Thus we obtain

$$
g_{H}(E)=2 E g_{\Delta}\left(E^{2}-3\right)
$$

For the density of states we obtain

$$
\rho_{H}=2|\epsilon| \rho_{\Delta}\left(\epsilon^{2}-3\right) .
$$

Actually, Equations (36) and (37) become completely obvious after we square the Hamiltonian (31) to get

$$
\hat{H}^{2}(\boldsymbol{k})=\left(\begin{array}{cc}
\epsilon_{\Delta}(\boldsymbol{k})+3 & 0 \\
0 & \epsilon_{\Delta}(\boldsymbol{k})+3
\end{array}\right) .
$$

The DOS is presented on Figure 3.

\section{Kagome Lattice}

The kagome lattice can be considered as a triangular lattice (which we consider to be identical to the lattice introduced above for the honeycomb lattice) with a basis of three lattice points. The Hamiltonian is

$$
\hat{H}=\sum_{n}\left[a_{n}^{\dagger}\left(b_{n+\delta_{3}}+b_{n-\delta_{3}}\right)+a_{n}^{\dagger}\left(c_{n+\delta_{2}}+c_{n-\delta_{2}}\right)+b_{n+\delta_{1}}^{\dagger}\left(c_{n+\delta_{1}}+c_{n-\delta_{1}}\right)+\text { H.c }\right],
$$

where $\delta_{i}=\boldsymbol{a}_{i} / 2$ are the vectors connecting the nearest neighbors.

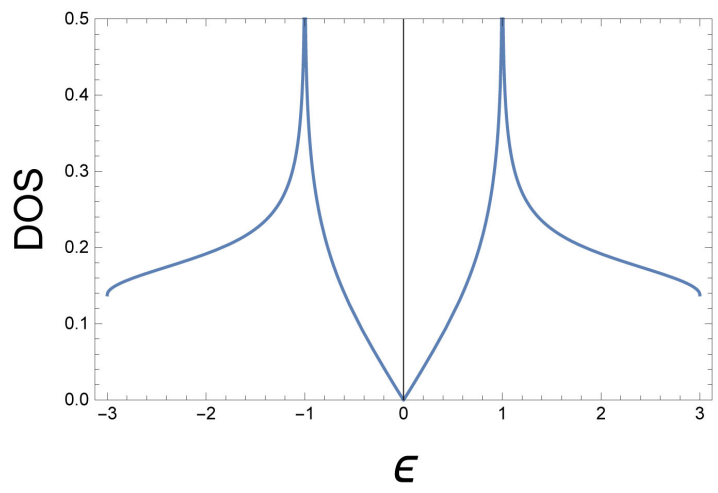

Figure 3. DOS for the honeycomb lattice. 
Going to wave vector representation we obtain

$$
\hat{H}=\sum_{\boldsymbol{k}} \Psi_{\boldsymbol{k}}^{\dagger} \hat{H}(\boldsymbol{k}) \Psi_{\boldsymbol{k}},
$$

where

$$
\hat{H}(\boldsymbol{k})=2\left(\begin{array}{ccc}
0 & \cos \left(\frac{k_{3}}{2}\right) & \cos \left(\frac{k_{2}}{2}\right) \\
\cos \left(\frac{k_{3}}{2}\right) & 0 & \cos \left(\frac{k_{1}}{2}\right) \\
\cos \left(\frac{k_{2}}{2}\right) & \cos \left(\frac{k_{1}}{2}\right) & 0
\end{array}\right) .
$$

The spectrum is found from the equation

$$
\operatorname{det}(\epsilon \hat{I}-\hat{H})=0 \text {. }
$$

Calculating the determinant and taking into account that $k_{1}+k_{2}+k_{3}=0$ we may present the dispersion equation as

$$
[\epsilon(\boldsymbol{k})+2]\left[\epsilon^{2}(\boldsymbol{k})-2 \epsilon(\boldsymbol{k})-X_{\boldsymbol{k}}+1\right]=0 .
$$

Thus we have a flat band $\epsilon_{F}(\boldsymbol{k})=-2$, and two dispersive bands

$$
\epsilon_{v}(\boldsymbol{k})=1+v \sqrt{X_{\boldsymbol{k}}} \text {. }
$$

The Green's function is

$$
g_{K}(E)=\frac{1}{(2 \pi)^{2}} \int_{-\pi}^{\pi} \int_{-\pi}^{\pi} \frac{\mathrm{d} k_{1} \mathrm{~d} k_{2}}{E+2}+\frac{1}{2 \pi^{2}} \int_{-\pi}^{\pi} \mathrm{d} k_{1} \int_{-\pi}^{\pi} \mathrm{d} k_{2} \frac{E-1}{E^{2}-2 E-2-\epsilon_{\Delta}(k)} .
$$

Comparing Equations (45) and (14) we obtain

$$
g_{K}(E)=\frac{1}{E+2}+2(E-1) g_{\Delta}\left[(E-1)^{2}-3\right]
$$

Hence

$$
\rho_{K}=\delta(\epsilon+2)+2|\epsilon-1| \rho_{\Delta}\left[(\epsilon-1)^{2}-3\right] .
$$

We see that apart from the $\delta$-function peak, the DOS for the Kagome lattice is the DOS for the honeycomb lattice shifted by 1 . The DOS is presented on Figure 4.

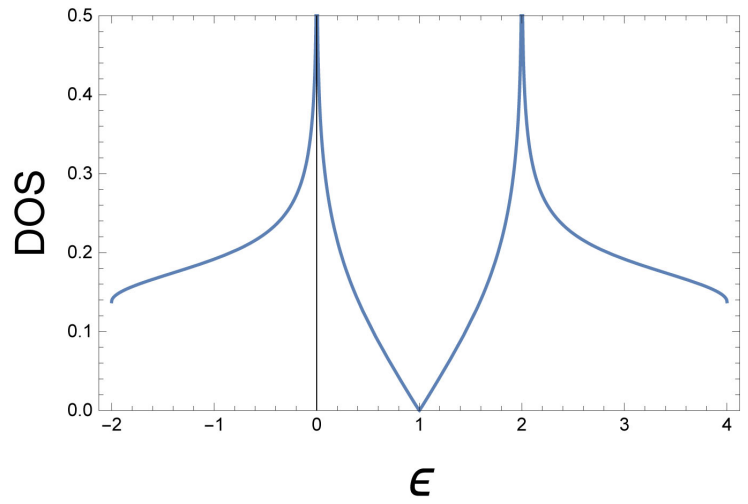

Figure 4. DOS for the kagome lattice. (The $\delta$-function peak is omitted). 


\section{Lieb Lattice}

Consider now the Lieb lattice with the Hamiltonian

$$
\hat{H}(\boldsymbol{k})=2\left(\begin{array}{ccc}
0 & \cos \left(\frac{k_{x}}{2}\right) & 0 \\
\cos \left(\frac{k_{x}}{2}\right) & 0 & \cos \left(\frac{k_{y}}{2}\right) \\
0 & \cos \left(\frac{k_{y}}{2}\right) & 0
\end{array}\right) .
$$

The spectrum of the Hamiltonian (48) is

$$
\epsilon_{F}(\boldsymbol{k})=0, \quad \epsilon_{v}(\boldsymbol{k})=2 v \sqrt{\cos ^{2}\left(\frac{k_{x}}{2}\right)+\cos ^{2}\left(\frac{k_{y}}{2}\right)} .
$$

The Green's function is

$$
g_{L}(E)=\frac{1}{(2 \pi)^{2}} \int_{-\pi}^{\pi} \int_{-\pi}^{\pi} \frac{\mathrm{d} k_{1} \mathrm{~d} k_{2}}{E}+\frac{1}{2 \pi^{2}} \int_{-\pi}^{\pi} \mathrm{d} k_{1} \int_{-\pi}^{\pi} \mathrm{d} k_{2} \frac{2 E}{E^{2}-4-\epsilon_{\square}(\boldsymbol{k})} .
$$

Comparing Equations (50) and (3) we obtain

$$
g_{L}(E)=\frac{1}{E}+2 E g_{\square}\left(E^{2}-4\right) .
$$

Hence

$$
\rho_{K}=\delta(\epsilon)+2|\epsilon| \rho_{\square}\left(\epsilon^{2}-4\right)=\delta(\epsilon)+\frac{|\epsilon|}{\pi^{2}} K\left(\sqrt{1-\frac{\left(\epsilon^{2}-4\right)^{2}}{16}}\right) .
$$

The DOS is presented on Figure 5.

\section{Conclusions}

To summarize, we have expressed lattice Green's functions and density of states for triangular and square lattice through the complete elliptic integral of the first kind in a uniform way. The Green's functions were calculated analytically as functions of energy on the part of real axis, and then continued analytically on the whole complex plane.

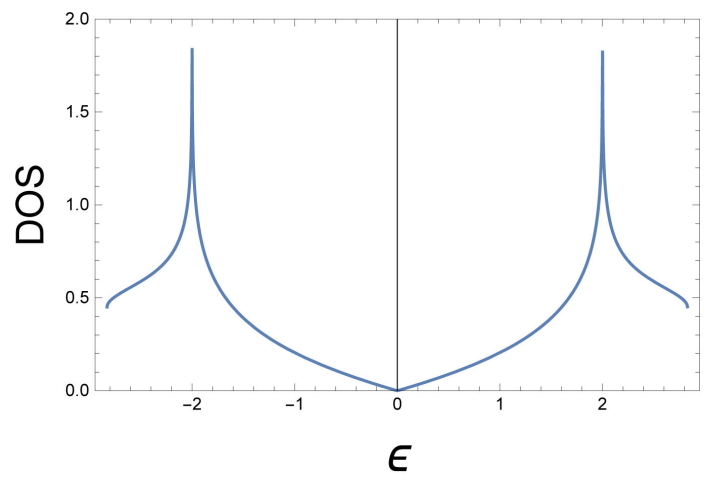

Figure 5. DOS for the Lieb lattice. (The $\delta$-function peak is omitted). 
We connected lattice Green's functions and density of states for honeycomb and kagome lattices with those of the triangular lattice, and Green's functions and density of states for Lieb lattice with those of square lattice. Exact expressions for lattice density of states ought to be useful in dynamical mean field theory calculations [19].

We have also shown that the well known results for the wave functions of electrons in the vicinity of the Dirac points in the honeycomb lattice are not connected with the nearest-neighbor hopping approximation, but follow from the symmetry of the model.

\section{Acknowledgements}

The work on this paper started during E.K. visit to Max-Planck-Institut für Physik komplexer Systeme in December of 2019 and January of 2020. E.K. cordially thanks the Institute for the hospitality extended to him during then and for all his previous visits. G.G. would like to acknowledge the support from the Air Force Research Laboratory (AFRL) through Grant No. FA9453-21-1-0046. The authors are grateful to V.O. Ananyev for attracting attention to the paper [22].

\section{Conflicts of Interest}

The authors declare no conflicts of interest regarding the publication of this paper.

\section{References}

[1] Komnik, A. and Heinze, S. (2017) Analytical Results for the Green's Functions of Lattice Fermions. Physical Review B, 96, Article ID: 155103.

https://doi.org/10.1103/PhysRevB.96.155103

[2] Varma, V.K. and Monien, H. (2013) Lattice Green's Functions for Kagome, Diced, and Hyperkagome Lattices. Physical Review E, 87, Article ID: 032109. https://doi.org/10.1103/PhysRevE.87.032109

[3] Katsura, S., Morita, T., Inawashiro, S., Horiguchi, T. and Abe, Y. (1971) Lattice Green's Function. Introduction. Journal of Mathematical Physics, 12, 892-895. https://doi.org/10.1063/1.1665662

[4] Economou, E.N. (2006) Green's Functions in Quantum Physics. 3rd Edition, Springer-Verlag, Berlin.

[5] Guttmann, A.J. and Prellberg, T. (1993) Staircase Polygons, Elliptic Integrals, Heun Functions, and Lattice Green Functions. Physical Review E, 47, R2233-R2236. https://doi.org/10.1103/PhysRevE.47.R2233

[6] Ziff, R.M. (1991) Flux to a Trap. Journal of Statistical Physics, 65, 1217-1233. https://doi.org/10.1007/BF01049608

[7] Barber, M.N. and Ninham, B.W. (1970) Random and Restricted Walks. Gordon and Breach, New York.

[8] Hughes, B.D. (1995) Random Walks and Random Environments. Volume 1: Random Walks. Clarendon, Oxford.

[9] Cserti, J. (2000) Application of the Lattice Green's Function for Calculating the Resistance of an Infinite Network of Resistors. American Journal of Physics, 68, 
896-906. https://doi.org/10.1119/1.1285881

[10] Sherafati, M. and Satpathy, S. (2011) RKKY Interaction in Graphene from Lattice Green's Function. Physical Review B, 83, Article ID: 165425.

https://doi.org/10.1103/PhysRevB.83.165425

[11] Parhizgar, F., Sherafati, M., Asgari, R. and Satpathy, S. (2013) Ruderman-Kittel-Kasuya-Yosida Interaction in Biased Bilayer Graphene. Physical Review B, 87, Article ID: 165429. https://doi.org/10.1103/PhysRevB.87.165429

[12] Zare, M. (2019) RKKY Plateau in Zero- and One-Dimensional Triangular Kagome Lattice Models.

[13] Roslyak, O., Gumbs, G., Balassis, A. and Elsayed, H. (2020) Effect of Magnetic Field and Chemical Potential on the RKKY Interaction in the $\alpha$-T $\mathrm{T}_{3}$ Lattice.

[14] Prudnikov, A.P., Brychkov, Yu.A. and Marichev, O.I. (1986) Integrals and Series, Vol. 2. Gordon and Breach Science Publishers, Amsterdam.

[15] Henyey, F.S. and Seshadri, V. (1982) On the Number of Distinct Sites Visited in 2D Lattices. The Journal of Chemical Physics, 76, 5530-5534. https://doi.org/10.1063/1.442908

[16] Hanisch, Th., Uhrig, G.S. and Muller-Hartmann, E. (1997) Lattice Dependence of Saturated Ferromagnetism in the Hubbard Model. Physical Review B, 56, 1396013982. https://doi.org/10.1103/PhysRevB.56.13960

[17] Moritz, B. and Schwalm, W. (2001) Triangle Lattice Green Functions for Vector Fields. Journal of Physics A: Mathematical and General, 34, 589-602. https://doi.org/10.1088/0305-4470/34/3/317

[18] Erdelyi, A. (1985) Higher Transcendental Functions, Vol. II. McGraw-Hill Book Company, Inc., New York.

[19] Georges, A., Kotliar, G., Krauth, W. and Rozenberg, M. (1996) Dynamical Mean-Field Theory of Strongly Correlated Fermion Systems and the Limit of Infinite Dimensions. Reviews of Modern Physics, 68, 13-125.

https://doi.org/10.1103/RevModPhys.68.13

[20] Guinea, F., Peres, N.M.R., Novoselov, K.S. and Geim, A.K. (2009) The Electronic Properties of Graphene A. H. Castro Neto. Reviews of Modern Physics, 81, 109-162. https://doi.org/10.1103/RevModPhys.81.109

[21] Landau, L.D. and Lifshitz, E.M. (1991) Quantum Mechanics. Pergamon Press, Oxford.

[22] Ananyev, V.O. and Ovchynnikov, M.I. (2017) On the Density of States of Graphene in the Nearest-Neighbor Approximation. Condensed Matter Physics, 20, Article ID: 43705. https://doi.org/10.5488/CMP.20.43705 


\section{Appendix A: Why the Wave Functions of Electrons in Honeycomb Lattice Define What They Are?}

The results for the wave functions of electrons in the vicinity of the Dirac points in the honeycomb lattice (Equations (A9) and (A10) below) are well known [20]. In this appendix we want to emphasise the fact that these results are not connected, as it is sometimes erroneously stated, with the nearest-neighbor hopping approximation, but follow from the symmetry of the model.

Looking at Equation (33) we understand that to find minimal value of $X_{\boldsymbol{k}}$ we should find minimal value of the sum of cosines of three numbers which add up to zero. This value is equal to $-3 / 2$ and is achieved when two of the numbers are $2 \pi / 3$ and the third number is $-4 \pi / 3$ (the point $K$ ), or when two of the numbers are $-2 \pi / 3$ and the third number is $4 \pi / 3$ (the point $\boldsymbol{K}^{\prime}$ ). The bands touch each other at these points.

The wave functions can be presented as

$$
\Psi_{v}(\boldsymbol{k})=\frac{1}{\sqrt{2}}\left(\begin{array}{c}
\mathrm{e}^{-i \tilde{\theta}_{\boldsymbol{k}} / 2} \\
v \mathrm{e}^{i \tilde{\theta}_{\boldsymbol{k}} / 2}
\end{array}\right),
$$

where

$$
\tilde{\theta}_{k}=\operatorname{Arg}\left(\sum_{i} \mathrm{e}^{i \delta_{i} \cdot k}\right)
$$

Making substitution

$$
\boldsymbol{k}=\boldsymbol{K}+\boldsymbol{q}
$$

expanding with respect to $\boldsymbol{q}$ and keeping only the linear terms we obtain

$$
X_{\boldsymbol{q}}=\frac{1}{2} \sum_{i}\left(\boldsymbol{a}_{i} \cdot \boldsymbol{q}\right)^{2}=\frac{3}{4} q^{2} .
$$

Thus we have the conic point in the spectrum.

In the same approximation

$$
\sum_{i} \mathrm{e}^{i \boldsymbol{\delta}_{i} \cdot \boldsymbol{k}}=\sum_{i} \mathrm{e}^{i \boldsymbol{\delta}_{i} \cdot \boldsymbol{K}}\left(\boldsymbol{\delta}_{i} \cdot \boldsymbol{q}\right)=(\boldsymbol{n}+\mathrm{im}) \cdot \boldsymbol{q},
$$

where

$$
\boldsymbol{n}=\sum_{i} \boldsymbol{\delta}_{i} \cos \left(\boldsymbol{\delta}_{i} \cdot \boldsymbol{K}\right), \boldsymbol{m}=\sum_{i} \boldsymbol{\delta}_{i} \sin \left(\boldsymbol{K} \boldsymbol{\delta}_{i}\right)
$$

One can easily check up that

$$
\boldsymbol{n} \perp \boldsymbol{m},|\boldsymbol{n}|=|\boldsymbol{m}| .
$$

Hence $\tilde{\theta}_{\boldsymbol{q}}=\theta_{\boldsymbol{q}}$, where $\theta_{\boldsymbol{q}}$ is the polar angle of $\boldsymbol{q}$, the $X$ axis being chosen in the direction of $n$. If we chose

$$
\begin{aligned}
& \delta_{1}=\frac{1}{2}\left(\frac{1}{\sqrt{3}}, 1\right), \quad \delta_{2}=\frac{1}{2}\left(\frac{1}{\sqrt{3}},-1\right), \\
& \boldsymbol{\delta}_{3}=-\left(\frac{1}{\sqrt{3}}, 0\right), \quad \boldsymbol{K}=\left(\frac{2 \pi}{\sqrt{3}}, \frac{2 \pi}{3}\right),
\end{aligned}
$$

then $X$ axis is in the direction of $\delta_{2}$. Hence Equation (A1) can be written as 


$$
\Psi_{v, \boldsymbol{K}}(\boldsymbol{q})=\frac{1}{\sqrt{2}}\left(\begin{array}{c}
\mathrm{e}^{-i \theta_{\boldsymbol{q}} / 2} \\
v \mathrm{e}^{i \theta_{\boldsymbol{q}} / 2}
\end{array}\right)
$$

For the $\boldsymbol{K}^{\prime}$ point, the wavefunctions are obtained from those in Equation (A9) by permutation of the sublattices

$$
\Psi_{v, K^{\wedge}}(q)=\Psi_{v, \boldsymbol{K}}^{*}(q) .
$$

The natural question appears: are Equations (A9) and (A10) connected to the simplest possible tight-binding model we have used? The answer is: No, these equations are general, and follow from the symmetry of the problem. In fact, the general tight-binding Hamiltonian for the honeycomb lattice is

$$
\hat{H}=\left(\begin{array}{cc}
\sum_{a} t^{\prime}(\boldsymbol{a}) \mathrm{e}^{i \boldsymbol{k} \cdot \boldsymbol{a}} & \sum_{a} t(\boldsymbol{a}+\boldsymbol{d}) \mathrm{e}^{i \boldsymbol{k} \cdot(\boldsymbol{a}+\boldsymbol{d})} \\
\sum_{\boldsymbol{a}} t^{*}(\boldsymbol{a}+\boldsymbol{d}) \mathrm{e}^{-i \boldsymbol{k} \cdot(\boldsymbol{a}+\boldsymbol{d})} & \sum_{a} t^{\prime}(\boldsymbol{a}) \mathrm{e}^{i \boldsymbol{k} \cdot \boldsymbol{a}}
\end{array}\right),
$$

where the summation is with respect to all lattice vectors $\boldsymbol{a}$, and $\boldsymbol{a}$ is some arbitrary, but fixed $\delta_{i}$. The selection rule for matrix elements [21] gives

$$
\sum_{\boldsymbol{a}} t(\boldsymbol{a}+\boldsymbol{\delta}) \mathrm{e}^{i \boldsymbol{K} \cdot(\boldsymbol{a}+\boldsymbol{\delta})}=0 .
$$

In fact, we are dealing with the product of two functions. The function $t(\boldsymbol{a}+\boldsymbol{\delta})$ realizes the unit representation of the point symmetry group $C_{3}$ (the full symmetry group of the inter-sublattice hopping is $C_{3 v}$, but the restricted symmetry is enough to prove the cancelation). As far as the function $\mathrm{e}^{i \boldsymbol{K} \cdot(\boldsymbol{a}+\boldsymbol{\delta})}$ is concerned, rotation of the lattice by the angle $2 \pi / 3$, say anticlockwise, is equivalent to rotation of the vector $\boldsymbol{K}$ in the opposite direction that is substitution of the three equivalent corners of the Brilluoin zone. Thus the exponent $\mathrm{e}^{i \boldsymbol{K} \cdot(\boldsymbol{a}+\boldsymbol{\delta})}$ realizes $x$-iy representation of the group $C_{3}$. Because each of the multipliers in Equation (A12) realizes different irreducible representation of the symmetry group, the matrix element is equal to zero. Simply speaking, at a point $\mathbf{K}$ the sublattices become decoupled, and this explains the degeneracy of the electron states in this point (these points) or, in other words, merging of the two branches of the single Brilouin zone.

Hence, for a general tight-binding model, the nondiagonal matrix element of the Hamiltonian (A11) is a linear function of $\boldsymbol{q}:(\boldsymbol{c}+i \boldsymbol{d}) \cdot \boldsymbol{q}$, invariant with respect to rotation of $\boldsymbol{q}$ by $2 \pi / 3$ up to a $\boldsymbol{q}$ independent constant. It immediately leads to the demands $|\boldsymbol{c}|=|\boldsymbol{d}|$, and $\boldsymbol{c} \perp \boldsymbol{d}$ and we recover Equation (A9). Equation (A10) follows from Equation (A9) because of invariance of the system with respect to mirror reflections. 